\title{
Rhinocerebral mucormycosis in a patient with diabetes type I presenting as ptosis and facial palsy report from Alborz hospital of Karaj from Iran
}

\begin{abstract}
Mucormycosis is an angioinvasive infection caused by ubiquitous filamentous fungi of the order mucorales. It is a rapidly progressive fatal infection mostly reported in susceptible individuals, such as those with poorly controlled diabetes or those with defects in phagocytic function. Rhino cerebral mucormycosis is the most common type of mucormycosis in diabetic patients. This case report describes a 27 -year-old woman with type1 diabetes mellitus presenting with Rhino cerebral mucormycosis. The patient presented with a history of ptosis and facial palsy, signs of ocular and cranial nerve involvement and also left unilateral facial edema, per orbital edema, epistaxis, otorrhagia and signs of mid brain and cavernous sinus thrombosis and at last depressed level of consciousness, she had hyperglycemia with diabetic ketoacidosis and rapidly developed hemiparesis progressing to quadriparesis and died within four days of ICU admission. The current report emphasizes the importance of having a high index suspicion when dealing with patients with diabetes presenting with facial palsy and pain or cellulites and prompt initiation of medical therapy along with surgical debridement for control of rhino cerebral mucormycosis.
\end{abstract}

Volume 5 Issue 2 - 2017

\author{
Leila Kheirkhah, Sadegh Asoubar,Amir \\ Hossein Abdi,Azam Mahmoudi \\ Alborz Hospital,Alborz University of Medical Science, Iran
}

Correspondence: Leila Kheirkhah,Alborz Hospital,Alborz University of Medical Science, Alborz, Iran, Tel 09128595978, Email asoubarsadegh@gmail.com〉

Received: October 01, 2017 | Published: October 09, 2017

Keywords: mucormycosis, diabetes type 1, facial palsy

Abbreviations: ROCM, rhino cerebral mucormycosis; HSCT, hematopoietic stein cell transplantation: PCR, polymerase chain reaction; LFABS, lipid formulations AMB

\section{Background}

Rhino cerebral mucormycosis (ROCM) is the most common form of mucormycosis in patient with diabetes mellitus. This is an uncommon disease which sometimes presents as symptoms of bacterial or viral sinusitis, otitis, facial palsy, ptosis and earache in a patient with uncontrolled diabetes. The disease presents with a diagnostic dilemma as initial presentation mimics facial or orbital cellulites and ptosis or signs of cavernous sinus thrombosis. Also it has a very rapidly and relentless progressive clinical course and is almost universally fatal if untreated. The authors decided to write up this case because of the need for high index of suspicion in treating patients with this disease. The "black of the palate is widely described as a hallmark of ROCM but the astute clinician will recognize earlier manifestations of this end stage lesion reflecting invasion of the palate.

\section{Case presentation}

A 27-year-old woman presented in the emergency department with chief complaint of vomiting, ptosis, facial palsy and right hemiparesis with subsequent development of quadriparesis followed by cranial nerve involvement and signs of Pons and mid brain stroke in the course of hospitalization. She was a known case of type I diabetes mellitus since the age of 12 and had been on regular and NPH insulin twice a day with poorly controlled blood sugars. At the time of admission the patient was able to walk with difficulty, suffering from facial palsy and right hemi paresis. She had limitation of eye movement in different directions. Blood pressure was $110 / 60 \mathrm{~mm} \mathrm{hg}$, pulse rate $80 / \mathrm{min}$, respiratory rate $20 / \mathrm{min}$, Blood sugar was $291 \mathrm{mg} / \mathrm{dl}$ and temperature $37^{\circ} \mathrm{C}$. On examination, she was conscious and aware of the environment. There was facial edema, more on the left side with Periorbital edema at the same side, ptosis without purulent discharge. At that time there was no proptosis of either eyes. She had signs of left cavernous sinus thrombosis and left carotid obstruction also there were obvious signs of cranial nerves 3,6 involvements. After 18 hours of hospitalization she went under mechanical ventilation as a result of severe respiratory failure. She had right hemiplegia 0.5 and complete ptosis of the right eye.

\section{Investigations}

A complete blood count on the day of presentation was 25000 polymorphs, $88 \%$ lymphocytes $10 \%$ and random blood sugar was $291 \mathrm{mg} / \mathrm{dl}, \mathrm{LDH} 1074 \mathrm{U} / \mathrm{L}, \mathrm{CPK} 90 \mathrm{U} / \mathrm{L}, \mathrm{Na} 136 \mathrm{mg} / \mathrm{ml}, \mathrm{K} 4.2 \mathrm{mg} /$ $\mathrm{ml}$. The serum creatinine level was $0.5 \mathrm{mg} / \mathrm{dl}$. The urine examination showed no pus cells but was positive for ketone bodies. Arterial blood gas analysis showed metabolic acidosis with $\mathrm{pH}$ of 7.33. The initial $\mathrm{CT}$ of the brain showed no signs of infection, infarction or bone destruction. No attempt was made to investigate signs of infection in paranasal sinus.

\section{Differential diagnosis}

In a patient presenting with facial pain or palsy. Facial and Periorbital edema, orbital or facial cellulites and signs of cavernous sinus thrombosis caused by bacterial pathogens (mostly staphylococcus aureus, also streptococcal and Gram-negative species) must be excluded. There are also some other mold infections, including aspergillosis, scedosporiosis, fusariosis and infections by the dematiaceous fungi (brown-pigmented soil organisms), can cause clinical syndrome, identical to mucormycosis. Histopathology examination and a positive culture permit definitive species identification. Since the preferred antifungal treatment differ (i.e., 
polygene's for the mucorales vs. expanded-spectrum triazoles for most septate molds), it is of great importance to distinguish them from each other. Phycomycetes form nonseptate, irregularly wide (6 to 50) fungal hyphae with frequent right angle branching whilst Aspergillus forms fruiting bodies and septate filaments ( 2 to 5 ) thick and (5 to 10) in invasive forms with branching at acute angles $\left(40^{\circ} \mathrm{C}\right)$ called dichotomous.

\section{Treatment}

The patient presented with features suggestive of midbrain and cavernous sinus thrombosis with a low level of ability to movement and after that the relentless decease of consciousness and respiratory function. Insulin infusion, hydration, antibiotics and heparin were promptly started for the patient. On day two in spite of treatment she developed bluish-black discoloration on the left side of her nose at that time biopsy samples were obtained and also Amphotericin B 1mg/kg/day was initiated for her based on a high index of suspicion without waiting for a microbiological diagnosis. Serum potassium and creatinine levels were being monitored during infusion of Amphotericin B. On the same day the patient developed hypernatremia, hypokalemia, polyuria and coagulopathy with multiple bleeding mucosal wounds in her mouth. Fluid and electrolyte and blood sugar disturbance were managed tightly. On day three the patient had Otorrhagia from the right ear and at that moment her family gave us a history of patient's earache two weeks ago. The discoloration of the face was spreading from left to right with patches of necrosis. Despite optimal antifungal therapy and prompt reversal of diabetic ketoacidosis, patient had a progressive deterioration and developed quadriplegia and died on the fourth day of admission because of refractory hypotension. Unfortunately we didn't have access to MRI or MR Venography so the cause of quadriparesis could not be elicited definitely. The focal neurological deficit could be either due to vascular structures involvement, or cavernous sinus and intracranial contents (especially cranial nerves of 3 and 5) lesions

\section{Discussion}

Mucormycosis represents a group of life-threatening infections caused by fungi of the order mucorales of the subphylum mucoromycotina. Infection caused by Mucorales is most accurately referred to as Mucormycosis, although the term zygomycosis may still be used by some sources. Mucormycosis is highly invasive and relentlessly progressive, resulting in higher rates of morbidity and mortality than many other infections. ${ }^{1}$ Mucormycosis is a member of the mucoraceae family including the absidia, mucor and rhizopus which may be found in decaying foods, in the soil, other organic matter, such as animal excreta and bread mould, nasal mucosa of normal person. The organisms are aerobic. ${ }^{1-3}$ In a case series from southern area of Iran of 162 patients with Mucormycosis of which $(18.5 \%)$ had DM as the second predisposing factor after leukemia. ${ }^{4}$ A high index of suspicion is critical for diagnosis, and early initiation of therapy often before confirmation of the diagnosis-is necessary to optimize outcomes. The mucorales are ubiquitous environmental fungi to which humans are constantly exposed. Infection occurs after inhalation of spores and low oxygen, high glucose, acidic environment and high iron levels facilitate the germination of the fungi. ${ }^{5}$ This fungi cause infection primarily in patients with diabetes mellitus, mostly poorly controlled who have defects in neutrophils and phagocytic functions, patients under glucocorticoid treatment, hematopoietic stein cell transplantation (HSCT), patients with elevated levels of free iron, because of supporting the fungal growth and also treatment with deferoxamine predisposes to the development of rapidly fatal disseminated Mucormycosis because deferoxamine enhances delivery of iron to mucorales. However, it rarely causes an infection in one who has an intact immune system. ${ }^{6}$ In some instances, infection with Mucormycosis for the first time could be seen even in a person without the history of hyperglycemia, as a matter of fact diabetes in a patient may have been unmasked by this infection or by recent glucocorticoid use. In some cases like severe bum, renal disease, malnutrition and treatment of graft- versus- host disease there is a high rate of susceptibility of mucorales infection. ${ }^{1,7}$ Mucormycosis can occur as isolated cutaneous or subcutaneous infection in immunologically normal individuals due to traumatic implantation of contaminated soil or vegetation (e.g., natural disasters or motor vehicle accidents) or nosocomial settings via IV catheters, SC injections or maceration of the skin by a moist dressing. ${ }^{4}$ Patients receiving antifungal prophylaxis with either itraconazole or voriconazole may be at increased risk of Mucormycosis. Actually, this patient typically present with disseminated mucormycosis which is the most lethal form of disease. Mucormycosis can be divided into at least six clinical categories: rhino-orbital-cerebral, pulmonary, cutaneous, gastro-intestinal, disseminated, and miscellaneous. Rhino-orbitalcerebral is the most common form of infection in patients with DKA, whilst pulmonary mucormycosis is seen in leukemic patients who are receiving chemotherapy and in patients undergoing (HSCT) ${ }^{8-10}$ Rhino-orbital-cerebral Mucormycosis occur in patients with diabetes, and rhizopus is the predominant pathogen, with the incidence of $90 \%$ of rhino-orbital-cerebral infections. ${ }^{1}$ The initial symptoms of rhinoorbital-cerebral mucormycosis are nonspecific and include eye or facial pain, numbness, conjunctiva suffusion, blurred vision and loss of vision (indicating retinal artery thrombosis), fever, leukocytosis, paranasal and ethmoid sinus involvement orbit and extra ocular muscles infection resulting proptosis and chemosis. In the course of infection vision loss and ophthalmoplegia suggesting development of infection to cavernous sinus and causing thrombosis and spreading to sphenoid sinus and cranial content, which are symptoms indicating a poor prognosis usually followed by reduced consciousness.,11 Infection from maxillofacial region can enter the cavernous sinus directly from valve less superior and inferior ophthalmic veins and also by the preneural invasion resulting disseminated (ROCM). ${ }^{1}$

Orbital swelling and facial cellulites are progressive. Black pus discharges from the necrotic palatine or nasal eschars are due to angioinvasive nature of the fungi and presenting poor prognosis of the disease. Cranial nerves 5 and 7 are the most commonly affected. Biopsy with histopathology examination- the ribbon like form of the fungus- remains the most sensitive and specific modality for definitive diagnosis. Culture studies are usually unsuccessful. ${ }^{12}$ Positive culture is seen in fewer than half of cases. That indicates low sensitivity of culture in diagnosis of mucormycosis. Polymerase chain reaction (PCR) is being studied but is not yet approved. Radiographic findings are helpful just in assessing the stages of the infection and often underestimate the extent of the disease. Most common finding on CT or MRI of the head or sinuses in rhino-orbital-cerebral Mucormycosis is sinusitis which is indistinguishable from bacterial sinusitis (II). ${ }^{1}$ MRI remains more sensitive $(80 \%)$ for detecting orbital and CNS involvement than is CT. once a patient with predisposing factor and signs of sinusitis or bluish-black discoloration of nose and palate is presented an astute physician should be aware of (ROCM). Tissue biopsy is necessary to confirm the diagnosis and visualizing 
the characteristic hyphae is the only definite way to diagnose. ${ }^{1}$ If mucormycosis is suspected, initial empirical therapy with a polygene's antifungal agent should be initiated while the diagnosis is being confirmed. Owing to completely different medications, it is important to distinguish Mucormycosis from other mold infection especially from aspergillosis. Successful treatment of Mucormycosis requires four steps: early diagnosis, considering to predisposing risk factors, surgical debridement and prompt antifungal therapy. ${ }^{12,13}$ Early diagnosis has improved survival rates. Since mucormycosis often invades blood vessels, inflammation, necrosis and thrombosis are the main characteristics and could result in poor penetration of antifungal agents to the affected area. Therefore, requires debridement of all necrotic tissues as a fundamental principle of treatment. ${ }^{1}$ The drug of choice for mucormycosis is amphotericin B which is a polyene. Lipid formulations of AMB which is shown as (LFABS) are significantly less nephrotoxic. Therefore they can be administered safely at higher doses, and are probably more effective. ${ }^{10}$ Liposomal AMB is preferred to AMB and also to amphotericin B lipid complex (ABLC). In some animal studies, results from treatment of (ROCM) by (LAMB), (AMB) were significantly better than that with (ABLC). ${ }^{14}$ In these recent days there is emphasizes on combination of antifungal therapy instead of single therapy. ${ }^{15}$ As a matter of fact the combination of (caspofungin) with (ABLC) has markedly improved survival of infected mice, compared with monotherapy. Echinocandins enhance exposure of B-glucan on the fungal surface, which results in immune stimulation and as a cause of improving outcomes of mucormycosis. ${ }^{10}$

\section{Conclusion}

A high index of suspicious is critical for diagnosis, and early initiation of therapy often before the information of the diagnosis is required. The most common form of mucormycosis in diabetes mellitus is rhino-orbital-cerebral, though in some instances presentation for mucormycosis may result in the first clinical recognition of diabetes. Mucorales invade blood vessels and cause necrosis of vessel walls and mycotic thrombi in immuno compromised patients. It is of great importance to distinguish between mucormycosis and aspergillosis, since the treatment of the kilter will exacerbate mucormycosis. HRCT is the best method for pulmonary Mucormycosis. The symptoms and signs of facial cellulites, sinusitis, cranial nerve involvement, toothache, earache, facial palsy and facial pain, ptosis, chemosis, black-bluish discoloration of face, signs of cavernous sinus thrombosis am strong predictors of RCOM. The best method for diagnosis is biopsy while other fungi similar to mucormycosis can be distinguished by positive culture. CT and MRI of the head have been having unreliable findings in diagnosis. But the latter has been more sensitive $80 \%$ than CT. Initial empirical therapy should be started on suspicion of mucormycosis.

\section{Acknowledgements}

None.

\section{Conflict of interest}

The author declares no conflict of interest.

\section{References}

1. Khodayari A, Mashhadi Abbas F, Nasabi Z, et al. Rhino-orbito cerebral mucormycosis in an otherwise healthy patient with no history of systemic disease: a case report and review of literature. J Dent Sch GYEAR. 2013;30(4):256-260.

2. Kwon Chung KJ. Taxonomy of fungi causing mucormycosis and entomophthoramycosis (zygomycosis) and nomenclature of the disease: molecular mycologic perspectives. Clin Infect Dis. 2012;54(1):S8-15.

3. Chakrabarti A. Cutaneous zygomycosis: major concerns. Indian J Med Res. 2010;131(6):739-742.

4. Sarvestani AS, Pishdad G, Bolandparvaz S. Predisposing factors for mucormycosis in patients with diabetes mellitus; an experience of 21 years in Southern Iran. Bull Emerg Trauma. 2013;1(4):164-170.

5. Mohammadi R, Nazeri M, Sayedayn SMA, et al. A successful treatment of rhinocerebral mucormycosis due to Rhizopus oryzae. J Res Med Sci. 2014;19(1):72-74.

6. Casqueiro J, Casqueiro J, Alves C. Infections in patients with diabetes mellitus: A review of pathogenesis. Indian $J$ Endocrinol Metab. 2012;16(7):27-36.

7. Peleg AY, Weerarathna T, McCarthy JS, et al. Common infections in diabetes: pathogenesis, management and relationship to glycaemic control. Diabetes Metab Res Rev. 2007;23(1):3-13.

8. Almyroudis N, Sutton D, Linden P, et al. Zygomycosis in solid organ transplant recipients in a tertiary transplant center and review of the literature. Am J Transplant. 2006;6(10):2365-2374.

9. Roden MM, Zaoutis TE, Buchanan WL, et al. Epidemiology and outcome of zygomycosis: a review of 929 reported cases. Clin Infect Dis. 2005;41(5):634-653.

10. Goldstein EJ, Spellberg B, Walsh TJ, et al. Recent advances in the management of mucormycosis: from bench to bedside. Clin Infect Dis. 2009;48(12):1743-1751.

11. Hosseini SM, Borghei P. Rhinocerebral mucormycosis: pathways of spread. Eur Arch Otorhinolaryngol. 2005;262(11):932-938.

12. Mohammadi R, Meidani M, Mostafavizadeh K, et al. Case series of rhinocerebral mucormycosis occurring in diabetic patients. Caspian $J$ Intern Med. 2015;6(4):243-246.

13. Singh V, Singh M, Joshi C, et al. Rhinocerebral mucormycosis in a patient with type 1 diabetes presenting as toothache: a case report from Himalayan region of India. BMJ Case Rep. 2013;2013:bcr2013200811.

14. Reed C, Bryant R, Ibrahim AS, et al. Combination polyene-caspofungin treatment of rhino-orbital-cerebral mucormycosis. Clin Infect Dis. 2008;47(3):364-371.

15. Lamaris GA, Lewis RE, Chamilos G, et al. Caspofungin-mediated $\beta$-glucan unmasking and enhancement of human polymorphonuclear neutrophil activity against Aspergillus and non-Aspergillus hyphae. J Infect Dis. 2008;198(2):186-192. 